\title{
A Convenient One-Pot Method for the Synthesis of $N$-Methoxy- $N$-methyl Amides from Carboxylic Acids
}

\author{
Joong-Gon Kim and Doo Ok Jang ${ }^{\dagger, *}$ \\ Biotechnology Division, Hanwha Chemical Research and Development Center, Daejeon 305-345, Korea \\ †Department of Chemistry, Yonsei University, Wonju 220-710, Korea. *E-mail: dojang@yonsei.ac.kr \\ Received September 30, 2009, Accepted November 6, 2009
}

Key Words: $N$-Methoxy- $N$-methyl amide, Weinreb amide, $\alpha$-Amino Weinreb amide, Trichloroacetonitrile, Triphenylphosphine

$N$-Methoxy- $N$-methyl amides, or Weinreb amides, ${ }^{1}$ have been widely used as versatile synthetic intermediates in organic syntheses. ${ }^{2}$ These amides serve as excellent acylating agents for organolithium or organomagnesium reagents and as robust aldehyde group equivalents. ${ }^{3}$ The utility of Weinreb amides has been extended to the preparation of $N$-protected amino aldehydes, useful intermediates for many chemoselective transformations in peptide chemistry. ${ }^{4}$

Several approaches for the synthesis of Weinreb amides have been reported, including aminocarbonylative coupling ${ }^{5}$ and Stille cross-coupling. ${ }^{6}$ A one-pot synthesis of $\alpha$-siloxy Weinreb amides from aldehydes using masked acyl cyanide reagents has been reported. ${ }^{7}$ Weinreb amides have also been prepared using 4,6-pyrimidyl urethane and Grignard reagents. ${ }^{8}$ Among various methods for the synthesis of Weinreb amides, direct conversion from carboxylic acids is the most attractive because there is no need to transform the carboxylic acids into activated derivatives first. These methods rely mainly on the in situ activation of carboxylic acid followed by coupling with $\mathrm{N}, \mathrm{O}$-dimethylhydroxylamine. Several carboxylic acid-activating reagents have been used for this purpose including $N, N$ '-dicyclohexylcarbodiimide, ${ }^{9}$ propylphosphonic anhydride/ $N$-ethylmorpholine, ${ }^{10} N$-benzotriazole derivatives, ${ }^{11}[1,3,5]$ triazine derivatives, ${ }^{12} S, S$-di(2-pyridyl)dithiocarbonate, ${ }^{13}$ 2-bromo-1-methylpyridinium iodide, ${ }^{14}$ Deoxo-Fluor fluorinating agent, ${ }^{15}$ and others. ${ }^{16}$ However, many of these methods have drawbacks, which include the use of toxic or expensive reagents, long reaction times, high reaction temperatures, low yields, multi-step reactions, or tedious work-up procedures.

We report a mild and convenient one-pot method for the synthesis of Weinreb amides from carboxylic acids employing trichloroacetonitrile (TCA) and triphenylphosphine (TPP). We first presumed that carboxylic acid chlorides formed in situ from carboxylic acids with a combination of TCA and TPP ${ }^{17}$ were transformed into the corresponding Weinreb amides by treatment with $\mathrm{N}, \mathrm{O}$-dimethylhydroxylamine in the presence of triethylamine (TEA) (Scheme 1).

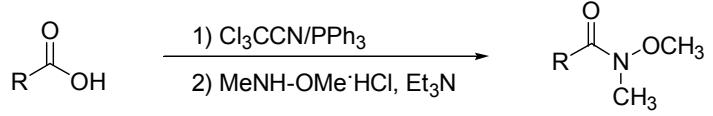

Benzoic acid was chosen as a model substrate to establish the optimal reaction conditions. The reaction parameters, reagent ratio, reaction solvent, and reaction temperature, were varied, and the results are summarized in Table 1 . When benzoic acid was treated with TCA and TPP in a ratio of $1.2: 1.2$, the corresponding Weinreb amide was obtained in $43 \%$ yield along with some unreacted benzoic acid (entry 1). However, when the ratio of TCA : TPP : benzoic acid was $2: 2: 1$, the amide was isolated in $92 \%$ yield (entry 2 ). An increase in the reagent ratio to $3: 3: 1$ did not further improve the reaction yield (entry 3 ). Likewise, increases in the amount of TEA, the reaction temperature, or the reaction time had no effect on the yield of the corresponding amide (entries 4-6). The effects of solvent on the reaction were also examined. Reactions carried out in $\mathrm{ClCH}_{2}$ $\mathrm{CH}_{2} \mathrm{Cl}, \mathrm{CH}_{3} \mathrm{CN}$, acetone, THF, EtOAc, or DMF afforded excellent yields of the corresponding amide (entries 7-12); reactions performed in ether or benzene produced somewhat lower yields (entries 13-14). These findings led us to conclude that the opti-

Table 1. Optimization of reaction conditions

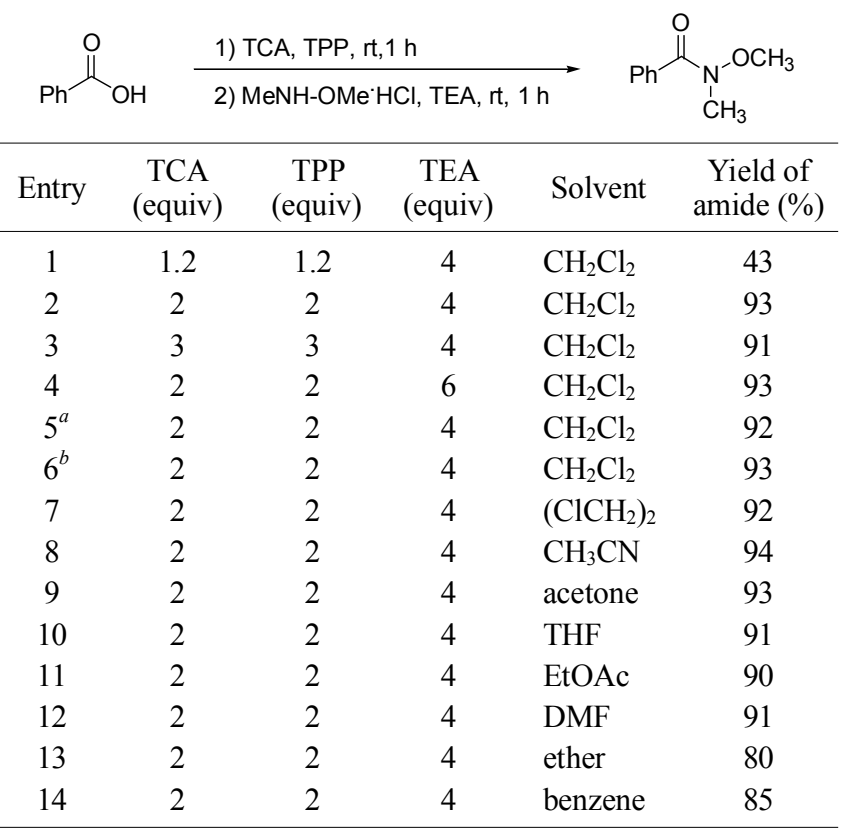

${ }^{a}$ The reaction was carried out at reflux in step $1 .{ }^{b}$ The reaction times were $2 \mathrm{~h}$ in step 1 and $2 \mathrm{~h}$ in step 2. 
Table 2. Formation of various Weinreb amides from carboxylic acids

\begin{tabular}{|c|c|c|c|}
\hline Entry & Carboxylic acid & Product & Yield (\%) \\
\hline 1 & & & $93^{16 a}$ \\
\hline 2 & & & $91^{13}$ \\
\hline 3 & & & $94^{13}$ \\
\hline 4 & & & $96^{16 b}$ \\
\hline 5 & & & $90^{11 \mathrm{c}}$ \\
\hline 6 & & & $88^{11 \mathrm{c}}$ \\
\hline 7 & & & $89^{13}$ \\
\hline $8^{b}$ & & & $83^{16 \mathrm{a}}$ \\
\hline $9^{b}$ & & & $79^{16 a}$ \\
\hline $10^{b}$ & & & $75^{16 a}$ \\
\hline 11 & & & $86^{13}$ \\
\hline 12 & & & $84^{13}$ \\
\hline 13 & & & 83 \\
\hline 14 & & & 86 \\
\hline 15 & & & $85^{13}$ \\
\hline $16^{b}$ & & & $80^{13}$ \\
\hline $17^{b}$ & & & $83^{13}$ \\
\hline $18^{b}$ & & $\mathrm{CH}_{3}$ & $82^{13}$ \\
\hline
\end{tabular}

${ }^{a}$ Typical experimental procedure: see text. ${ }^{b}$ The reaction was stirred for $2 \mathrm{~h}$ in step 2.
Table 3. Formation of various $N$-Fmoc $\alpha$-amino Weinreb amides from $\alpha$-amino acids ${ }^{a}$

\begin{tabular}{clll}
\hline Entry & \multicolumn{1}{c}{$\alpha$-Amino Acid } & \multicolumn{1}{c}{$\alpha$-Amino Weinreb Amide } & $\begin{array}{c}\text { Yield } \\
(\%)\end{array}$ \\
\hline 1 & $N$-Fmoc-L-Gly-OH & $N$-Fmoc-L-Gly-N $\left(\mathrm{OCH}_{3}\right) \mathrm{CH}_{3}$ & $89^{20}$ \\
2 & $N$-Fmoc-L-Ala-OH & $N$-Fmoc-L-Ala-N $\left(\mathrm{OCH}_{3}\right) \mathrm{CH}_{3}$ & $85^{20}$ \\
3 & $N$-Fmoc-L-Val-OH & $N$-Fmoc-L-Val-N $\left(\mathrm{OCH}_{3}\right) \mathrm{CH}_{3}$ & $83^{20}$ \\
4 & $N$-Fmoc-L-Leu-OH & $N$-Fmoc-L-Leu-N $\left(\mathrm{OCH}_{3}\right) \mathrm{CH}_{3}$ & $86^{20}$ \\
$5^{b}$ & $N$-Fmoc-L-Ile-OH & $N$-Fmoc-L-Ile-N $\left(\mathrm{OCH}_{3}\right) \mathrm{CH}_{3}$ & $80^{20}$ \\
$6^{b}$ & $N$-Fmoc-L-Met-OH & $N$-Fmoc-L-Met-N $\left(\mathrm{OCH}_{3}\right) \mathrm{CH}_{3}$ & $78^{20}$ \\
\hline
\end{tabular}

${ }^{a}$ THF was used instead of $\mathrm{CH}_{2} \mathrm{Cl}_{2} .{ }^{b}$ The reaction was stirred for $2 \mathrm{~h}$ in step 2 .

mal reaction conditions were TCA ( 2 equiv), TPP ( 2 equiv), and TEA (4 equiv) in $\mathrm{CH}_{2} \mathrm{Cl}_{2}$ at room temperature.

We applied the optimized reaction conditions to the preparation of other Weinreb amides. As shown in Table 2, the reaction was general for a wide range of carboxylic acids. As compared to aliphatic carboxylic acids, aromatic carboxylic acids afforded the corresponding amides in higher isolated yields (entries 1-7 $v s$. entries 11-13). Aromatic carboxylic acids with an electronwithdrawing group appear to be more reactive than aromatic carboxylic acids with an electron-donating group (entries 2-4). For instance, the reaction with $p$-methoxy benzoic acid required $1.5 \mathrm{~h}$ for the second step whereas the reaction with $p$-nitro benzoic acid took $0.5 \mathrm{~h}$. Heteroaromatic carboxylic acids are also good substrates for the reaction (entries 5-7). Stericallyhindered carboxylic acids were converted smoothly into the corresponding amides in good yields (entries 8-10). Aliphatic carboxylic acids containing olefin, chloro, carboethoxy, and benzoyl functional groups were also transformed into the corresponding amides in good yields, and the functional groups were unaffected by the reaction conditions (entries 14-18).

Next, we applied this new method to the synthesis of Fmocprotected $\alpha$-amino Weinreb amides. Various $N$-Fmoc- $\alpha$-amino acids were subjected to the reaction conditions to afford the corresponding Weinreb amides in $78-89 \%$ isolated yields. There were no signs of racemization or deblocking of the Fmoc group under the reaction conditions, based on analysis of the reaction mixtures by ${ }^{1} \mathrm{H}$ NMR and HPLC. ${ }^{18-19}$

In summary, we have developed a mild and convenient method for one-pot synthesis of Weinreb amides from carboxylic acids. The process is general for the preparation of Weinreb amides from a variety of carboxylic acids. The reaction was also applicable to the preparation of $\alpha$-amino Weinreb amides and proceeded without deprotection of the $N$-Fmoc protecting group or racemization of the stereogenic centers.

\section{Experimental Section}

Typical experimental procedure. To a mixture of benzoic acid $(122 \mathrm{mg}, 1.0 \mathrm{mmol})$ and triphenylphosphine $(525 \mathrm{mg}, 2.0$ $\mathrm{mmol})$ in dry $\mathrm{CH}_{2} \mathrm{Cl}_{2}(3 \mathrm{~mL})$ under argon at room temperature, trichloroacetonitrile $(0.2 \mathrm{~mL}, 2.0 \mathrm{mmol})$ was added dropwise. After $1 \mathrm{~h}, \mathrm{~N}, \mathrm{O}$-dimethylhydroxylamine. $\mathrm{HCl}(98 \mathrm{mg}, 1.0 \mathrm{mmol})$ and triethylamine $(0.56 \mathrm{~mL}, 4 \mathrm{mmol})$ were added to the mixture. The reaction mixture was stirred for another $1 \mathrm{~h}$ at room tem- 
perature while monitoring with TLC. When the reaction was complete, the mixture was diluted with $\mathrm{CH}_{2} \mathrm{Cl}_{2}(4 \mathrm{~mL})$ and was washed with saturated $\mathrm{NaHCO}_{3}$ solution $(3 \mathrm{~mL})$ and water $(3 \mathrm{~mL})$. The organic layer was dried over anhydrous $\mathrm{MgSO}_{4}$. After filtration, the solvent was removed, and the residue was purified by column chromatography on silica gel (hexanes/ EtOAc, $4: 1)$ to give $N$-methoxy- $N$-methyl benzamide (154 $\mathrm{mg}, 93 \%$ ).

2-Cyclopentyl- $\boldsymbol{N}$-methoxy- $\boldsymbol{N}$-methyl acetamide. ${ }^{1} \mathrm{H}$ NMR $\left(300 \mathrm{MHz}, \mathrm{CDCl}_{3}\right) \delta 1.16(\mathrm{~m}, 2 \mathrm{H}), 1.59(\mathrm{~m}, 4 \mathrm{H}), 1.84(\mathrm{~m}, 2 \mathrm{H})$, $2.29(\mathrm{~m}, 1 \mathrm{H}), 2.44(\mathrm{~d}, J=7.5 \mathrm{~Hz}, 2 \mathrm{H}), 3.18(\mathrm{~s}, 3 \mathrm{H}), 3.68(\mathrm{~s}$, $3 \mathrm{H}) ;{ }^{13} \mathrm{C} \mathrm{NMR}\left(75 \mathrm{MHz}, \mathrm{CDCl}_{3}\right) \delta 32.7,33.0,33.2,36.7,38.4$, 61.7, 174.9. Anal. Calcd for $\mathrm{C}_{9} \mathrm{H}_{17} \mathrm{NO}_{2}: \mathrm{C}, 63.13 ; \mathrm{H}, 10.01 ; \mathrm{N}$, 8.18. Found: C, 63.51; H, 10.25; N, 8.29.

2,2,2-Trichloro-N-methoxy-N-methyl acetamide. ${ }^{1} \mathrm{H}$ NMR $\left(300 \mathrm{MHz}, \mathrm{CDCl}_{3}\right) \delta 3.38(\mathrm{~s}, 3 \mathrm{H}), 3.82(\mathrm{~s}, 3 \mathrm{H}) ;{ }^{13} \mathrm{C} \mathrm{NMR}(75$ $\left.\mathrm{MHz}_{2} \mathrm{CDCl}_{3}\right) \delta 35.5,61.3,92.1,160.5$. Anal. Calcd for $\mathrm{C}_{4} \mathrm{H}_{6}$ $\mathrm{Cl}_{3} \mathrm{NO}_{2}$ : C, 23.27; H, 2.93; N, 6.78. Found: C, 23.63; H, 3.01; $\mathrm{N}, 6.64$.

Acknowledgments. This work was supported by the Center for Bioactive Molecular Hybrids.

\section{References}

1. Nahm, S.; Weinreb, S. M. Tetrahedron Lett. 1981, 22, 3815.

2. (a) Balasubramaniam, S.; Aidhen, I. S. Synthesis 2008, 23, 3707. (b) Banwell, M.; Edwards, A.; Smith, J.; Hame, E.; Verdier-Pinard, P. J. Chem. Soc., Perkin Trans 1 2000, 1497. (c) Perez, M.; del Pozo, C.; Reyes, F.; Rodriguez, A.; Francesch, A.; Echavarren, A. M.; Cuevas, C. Angew. Chem., Int. Ed. 2004, 43, 1724. (d) Mulzer, J.; Berger, M. J. Org. Chem. 2004, 69, 891. (e) Zanatta, S. D.; White, J. M.; Rizzacasa, M. A. Org. Lett. 2004, 6, 1041. (f) Davis, F. A.; Prasad, K. R.; Nolt, M. B.; Wu, Y. Z. Org. Lett. 2003, 5, 925. (g) Evans, D. A.; Connell, B. T. J. Am. Chem. Soc. 2003, 125, 10899. (h) Vanderwal, C. D.; Vosburg, D. A.; Weiler, S.; Sorensen, E. J. J. Am. Chem. Soc. 2003, 125, 5393.

3. (a) Huckins, J. R.; De Vicente, J.; Rychnovsky, S. D. Org. Lett. 2007, 9, 4757. (b) Gopalarathnam, A.; Nelson, S. G. Org. Lett. 2006, 8, 7. (c) Schuppan, J.; Ziemer, B.; Koert, U. Tetrahedron Lett. 2000, 41,621 .

4. (a) Gomez-Monterrey, I, M.; Campiglia, P.; Bertamino, A.; Aqui- no, C.; Mazzoni, O.; Diurno, M. V.; Iacovino, R.; Saviano, M.; Sala, M.; Novellino, E.; Grieco, P. Eur. J.Org. Chem. 2008, 11, 1983. (b) Gomez-Monterrey, I. M.; Campiglia, P.; Bertamino, A.; Mazzoni, O.; Diurno, M. V.; Novellino, E.; Grieco, P. Tetrahedron 2006, 62, 8083. (c) Grieco, P.; Campiglia, P.; Gomez-Monterrey, I.; Novellino, E. Tetrahedron Lett. 2002, 43, 6297. (d) Wu, Y.; Xu, J.-C. Tetrahedron 2001, 57, 8107. (e) Owens, T. D.; Araldi, G.-L.; Nutt, R. F.; Semple, J. E. Tetrahedron Lett. 2001, 42, 6271.

5. (a) Deagostino, A.; Karini, P.; Occhiato, E. G.; Pizzuto, L.; Prandi, C.; Venturello, P. J. Org. Chem. 2008, 73, 1941. (b) Martinelli, J. R.; Freckmann, D. M. M.; Buchwald, S. L. Org. Lett. 2006, 8, 4843. (c) Aungst, R. A.; Funk, R. L. J. Am. Chem. Soc. 2001, 123, 9455. 6. Murakami, M.; Hoshino, Y.; Ito, H.; Ito, Y. Chem. Lett. 1998, 163.

7. Nemoto, H.; Ma, R.; Moriguchi, H.; Kawamura, T.; Kamiya, M.; Shibuya, M. J. Org. Chem. 2007, 72, 9850.

8. Lee, J. I. Bull. Korean Chem. Soc. 2007, 28, 695.

9. (a) Gibson, C. L.; Handa, S. Tetrahedron-Asymmetry 1996, 7, 1281. (b) Braun, M.; Waldmuller, D. Synthesis 1989, 856.

10. (a) Dechantsreiter, M. A.; Burkhart, F.; Kessler, H. Tetrahedron Lett. 1998, 39, 253. (b) Oppolzer, W.; Cunningham, A. F. Tetrahedron Lett. 1986, $27,5467$.

11. (a) Katritzky, A. R.; Yang, H.; Zhang, S.; Wang, M. ARKIVOC 2002, 39. (b) Shreder, K.; Zhang, L.; Goodman, M. Tetrahedron Lett. 1998, 39, 221. (c) Maugras, I.; Poncet, J.; Jouin, P. Tetrahedron 1990, 46, 2807.

12. De Luca, L.; Gaicomelli, G.; Taddei, M. J. Org. Chem. 2001, 66, 2534.

13. Lee, J. I.; Park, H. Bull. Korean. Chem. Soc. 2001, 22, 421.

14. Sibi, M. P.; Stressman, C. C.; Schultz, J. A.; Christensen, J. W.; Lu, J.; Marvin, M. Synth. Commun. 1995, 25, 1255.

15. (a) White, J. M.; Tunoori, A. R.; Turunen, B. J.; Georg, G. I. J. Org. Chem. 2008, 73, 1941. (b) Tunoori, A. R.; White, J. M.; Georg, G. I. Org. Lett. 2000, 2, 4091.

16. (a) Han, K.-D.; Kim, M. Lett. Org. Chem. 2007, 4, 20. (b) Woo, J. C. S.; Fenster, E. Dake, G. R. J. Org. Chem. 2004, 69, 8984. (c) Kim, M.; Lee, H.; Han, K.-J.; Kay, K.-Y. Synth. Commun. 2003, 33, 4013. (d) Davies, D. T.; O’Hanlon, P. J. Synth. Commun. 1988, 18,2273

17. Jang, D. O.; Park, D. J.; Kim, J. Tetrahedron Lett. 1999, 40, 5323.

18. Chiral HPLC analysis for $N$-Fmoc-L-Ala-N( $\left(\mathrm{OCH}_{3}\right) \mathrm{CH}_{3}:[\alpha]_{\mathrm{D}}^{25}=$ $-11.1(\mathrm{c}=1.0, \mathrm{MeOH})\left(\right.$ lit. $\left.^{19}:[\alpha]_{\mathrm{D}}^{25}=-10.2(\mathrm{c}=1.0, \mathrm{MeOH})\right)$ Diacel Chiralcel OC; $n$-hexane/IPA $(90 / 10, \mathrm{v} / \mathrm{v})$; flow rate $=1.0$ $\mathrm{mL} / \mathrm{min}$; UV $(\lambda=254 \mathrm{~nm})$; retention time for L-form $=7.8 \mathrm{~min}$; retention time for $\mathrm{D}$-form $=9.5 \mathrm{~min}$.

19. Bailén, M. A.; Chinchilla, R; Dodsworth, D. J.; and Nájera, C. Tetrahedron Lett. 2001, 42, 5013.

20. Sureshbabu, V. V.; Hemantha, H. P. ARKIVOC 2008, 243. 